\title{
Fatores que afetam o desempenho de cordeiros Santa Inês puros e cruzados do nascimento ao desmame
}

\author{
Factors that affect the performance from birth to weaning on purebred and crossbred \\ Santa Ines lambs
}

\author{
KORITIAKI, Natália Albieri ${ }^{1 *}$; RIBEIRO, Edson Luis de Azambuja²; SCERBO, \\ Danielle Clivati ${ }^{1}$; MIZUBUTI, Ivone Yurika ${ }^{2}$; SILVA, Leandro das Dores Ferreira $\mathrm{da}^{2}$; \\ BARBOSA, Marco Aurélio Alves de Freitas²; SOUZA, Cícero Leandro de ${ }^{1}$; PAIVA, \\ Fernando Henrique Pereira de ${ }^{1}$
}

${ }^{1}$ Universidade Estadual de Londrina, Programa de Pós-graduação em Ciência Animal, Londrina, Paraná, Brasil. ${ }^{2}$ Universidade Estadual de Londrina, Departamento de Zootecnia, Londrina, Paraná, Brasil.

*Endereço para correspondência: nataliaalbieri@hotmail.com

\section{RESUMO}

Objetivou-se avaliar os efeitos ambientais (idade da mãe ao parto, tipo de nascimento, sexo do cordeiro e ano de nascimento) e os grupos genéticos sobre o peso e medidas morfométricas de cordeiros, ao nascimento e ao desmame; testar regressões para predizer peso por meio das medidas e descrever o tipo de crescimento das medidas. Os filhos de ovelhas mais jovens (dois dentes) apresentaram médias menores para todas as características, exceto altura ao desmame. As médias de peso ao nascimento variaram de 3,34 \pm $0,14 \mathrm{~kg}$ nos filhos de ovelhas de dois dentes a 3,94 $\pm 0,09 \mathrm{~kg}$ nos filhos de ovelhas de oito dentes, e as de peso ao desmame variaram de $12,19 \pm 0,72 \mathrm{~kg}$ nos filhos de ovelhas de dois dentes a 14,79 $\pm 0,62$ nos filhos de ovelhas de seis dentes. Os cordeiros nascidos de partos simples apresentaram maiores médias que os gêmeos. As médias de peso ao nascer foram de $3,24 \pm 0,11 \mathrm{~kg}$ a $4,08 \pm 0,06 \mathrm{~kg}$ e para peso ao desmame foram de $11,18 \pm 0,59 \mathrm{~kg}$ a $15,81 \pm 0,35 \mathrm{~kg}$, as médias menores foram dos cordeiros gêmeos e as maiores dos cordeiros simples. O grupo genético influenciou o peso, o comprimento e a altura ao nascimento, os Santa Inês puros apresentaram médias maiores que os $1 / 2$ Santa Inês. O ano de nascimento influenciou todas as características. Todas as correlações entre pesos e medidas foram significativas, e assim foi possível prever o peso em função das medidas.

Palavras-chave: crescimento alométrico, morfometria corporal, ovinos, peso corporal

\section{SUMMARY}

The objective of this study was to evaluate the environmental effects (age of dam, type of birth, sex of the lamb and year of birth) and genetic groups on the weight and morphometric measurements of lamb at birth and at weaning; test regressions for predicting weight of lambs by the measurements and describe the type of growth of the measures. Lambs of young ewes (2 teeth) had lower average for all characteristics except height at weaning. The average birth weight ranged from $3.34 \pm 0.14 \mathrm{~kg}$ in the offspring of two teeth ewes to $3.94 \pm$ $0.09 \mathrm{~kg}$ in the lambs of eight teeth ewes, and for weaning weight ranged from $12.19 \pm 0.72 \mathrm{~kg}$ in the offspring of two teeth ewes, and $14.79 \pm$ 0.62 in the lambs of six teeth ewes. The single birth lambs had highter averages than the twins. Means for birth weight ranged from $3.24 \pm$ $0.11 \mathrm{~kg}$ to $4.08 \pm 0.06 \mathrm{~kg}$ and for weaning weight from $11.18 \pm 0.59 \mathrm{~kg}$ to $15.81 \pm 0.35 \mathrm{~kg}$, the averages were lower for twins and greater for single lambs. The genetic group affected the weight and the length and height at birth, where purebred Santa Inês lambs presented greater averages than $1 / 2$ Santa Inês lambs. The year of birth influenced all traits. All correlations between weights and measures were significant. It is possible to predict the weight on the basis of measures.

Keywords: allometric growth, body morphometry, body weight, sheep 


\section{INTRODUÇ̃̃O}

A produção e o consumo de carne ovina no Brasil têm crescido e estimulado a realização de pesquisas, por meio das quais objetiva-se melhorar os sistemas de produção mediante práticas de manejo, alimentação, cruzamentos e seleção dos indivíduos ou raças, a fim de oferecer um produto de melhor qualidade a um mercado exigente (MACMANUS et al., 2003).

O crescimento dos animais constitui-se uma peça fundamental nas cadeias produtivas e nas indústrias de carne ovina, e o conhecimento dos fatores que afetam o bom desenvolvimento dos cordeiros é necessário para se atingir uma melhor eficiência da produção (SANTOS et al., 2001). Segundo Rocha et al. (2009) o sexo do cordeiro, o tipo de nascimento, grupo genético e o ano de nascimento são fatores que podem influenciar no desempenho dos animais. O fenótipo de um animal para tamanho e conformação é resultado do seu potencial genético, dos efeitos maternos e de outras influências do ambiente. As mensurações corporais em idade precoce têm influência direta materna e são importantes para estimar $o$ crescimento animal (MANDAL et al., 2008). As mensurações têm sido bem utilizadas para avaliar o desempenho e caracterizar os grupos genéticos, são facilmente obtidas e variam de acordo com o peso do animal (SOWAND \& SOBOLA, 2008).

A raça Santa Inês, de grande importância no cenário nacional é nativa do Nordeste brasileiro e tem demonstrado ser muito promissora para a produção de carne (GUEDES et al., 2005). É bem utilizada como linhagem materna para produção de cordeiros devido a sua maior rusticidade, prolificidade, menor estacionalidade reprodutiva e menor tamanho quando comparada a raças especializadas de corte (MALHADO et al., 2008).

As partes do corpo estudadas alometricamente podem explicar as diferenças quantitativas produzidas nas distintas fases da vida do animal, proporciona uma descrição quantitativa da relação parte/todo, e mesmo que não se registre detalhes, ela é relevante porque reduz toda a informação a um só valor (SANTOS et al., 2001).

Diante do exposto, objetivou- se com a realização deste trabalho avaliar a influência do ano de nascimento, tipo de nascimento, sexo da cria, grupo genético, peso e idade da mãe ao parto em relação ao desempenho e características morfométricas dos cordeiros ao nascimento e ao desmame (70 dias de idade). Testar regressões lineares e não lineares para predizer peso vivo por meio das medidas corporais e descrever o tipo de crescimento das medidas do corpo no período estudado.

\section{MATERIAL E MÉTODOS}

Os dados foram provenientes do setor de ovinocultura da Fazenda Escola da Universidade Estadual de Londrina (UEL) e coletados em três anos (2008; 2009 e 2010). A unidade experimental está localizada a $23^{\circ} 23^{\prime} \mathrm{S}$ de latitude e $51^{\circ} 11^{\prime} \mathrm{W}$ de longitude.

Os animais permaneceram em condições similares de manejo. As ovelhas e os cordeiros foram mantidos em pastagem de Coastcross (Cynodon dactylon (L.) Pers), com sal mineralizado à disposição em cochos apropriados e receberam como suplementação silagem de sorgo e concentrado durante os períodos de inverno. $\mathrm{O}$ manejo sanitário foi $\mathrm{o}$ usual da propriedade, com controle de verminose realizado conforme 
resultados de exame de fezes. Os cordeiros foram desmamados aos 70 dias de idade.

Foram analisados 102 cordeiros Santa Inês puros e 48 cruzados ( $1 / 2$ Santa Inês $+1 / 2$ SRD). Para as análises gerais dos efeitos ambientais e de grupos genéticos foram utilizadas as características de peso e medidas corporais realizadas ao nascimento e ao desmame, e para as análises do tipo de crescimento (alométrico ou isométrico) foram utilizadas mensurações realizadas do nascimento ao desmame a cada 14 dias, em um total de seis mensurações por característica.

As mensurações de morfometria corporal foram realizadas com o auxilio de fita métrica, com o animal mantido em posição correta de aprumos. A altura da cernelha foi medida entre o ponto mais alto da região interescapular e o solo. O comprimento corporal foi medido da cernelha até a parte caudal da tuberosidade isquiática. $\mathrm{O}$ perímetro torácico foi medido na circunferência externa da cavidade torácica, junto às axilas.

As características estudadas foram submetidas à análise de variância, e tiveram como variáveis independentes o grupo genético, tipo de nascimento (simples ou gemelar), sexo (macho ou fêmea), a idade da mãe ao parto (dois, quatro, seis ou oito dentes incisivos permanentes), ano de nascimento do cordeiro e como covariável peso da mãe ao parto (SAS, 1994). As interações entre as variáveis independentes foram desconsideradas na análise final por não apresentarem significância $(\mathrm{P}>0,05)$. As médias para grupo genético, idade da mãe ao parto e ano de nascimento foram comparadas pelo teste Tukey, e as demais pelo teste F. O modelo linear utilizado nas análises das características de pesos e medidas morfométricas foi:

$Y_{i j k l m n}=\mu+I_{i}+S_{j}+G_{k}+T_{l}+A_{m}+P_{n}+\varepsilon_{i j k l m !}$ em que $Y_{i j k l m}=$ variáveis dependentes (pesos ou medidas corporais); $\mu=$ média geral; $I_{i}=$ efeito da idade da mãe ao parto, sendo $\mathrm{i}=$ dois, quatro, seis ou oito dentes; $S_{j}=$ efeito do sexo do cordeiro, sendo $\mathrm{j}=$ macho ou fêmea; $G_{k}=$ efeito do grupo genético, sendo $\mathrm{k}=$ Santa Inês ou $1 / 2$ Santa Inês; $T_{l}=$ efeito do tipo de nascimento, sendo $\mathrm{l}=$ simples ou gemelar; $A_{m}=$ efeito do ano de nascimento do cordeiro, sendo $\mathrm{m}=$ 2008, 2009 ou 2010; $P_{n}=$ efeito do peso da ovelha ao parto (covariável); $\varepsilon_{i j k l m}=$ erro aleatório associado a cada observação.

Foram testadas análises de regressões não lineares e lineares para predição do peso vivo dos cordeiros ao nascimento e ao desmame em função das mensurações de morfometria corporal com a utilização do procedimento REG, stepwise do SAS (1994). Os seguintes modelos foram utilizados:

$Y=a+b X$....(Linear)

$Y=a+b_{1} X_{1}+\ldots+b_{n} X_{n} \ldots$ (Linear múltipla)

$Y=a+X^{b} \ldots$ (Alométrica)

em que $Y=$ peso vivo; $a=$ intercepto; $X$ $=$ medidas corporais; $b=$ coeficiente de regressão de $Y$ em $X ; n=$ número de mensurações corporais. A aderência do modelo foi avaliada por meio do coeficiente de determinação $\left(\mathrm{R}^{2}\right)$ das equações.

Para a determinação do tipo de crescimento das medidas corporais foi utilizada a equação "Y=aX" de Huxley (1932), por meio da transformação logarítmica em uma regressão linear simples: "ln $Y=\ln a+b(\ln X) "$, em que "Y" foi considerado como sendo o peso do animal, " $\mathrm{X}$ " o tamanho das partes do corpo do animal, "a" intercepção do logaritmo da regressão linear sobre "Y" (antilogaritmo de "a") e "b" o coeficiente de crescimento relativo, ou o coeficiente de alometria, que é a velocidade relativa de crescimento de 
Rev. Bras. Saúde Prod. Anim., Salvador, v.13, n.1, p.258-270 jan/mar, 2012 http://www.rbspa.ufba.br ISSN 15199940

"Y" em relação a " $\mathrm{X}$ ".

As análises para obtenção dos coeficientes alométricos foram realizados por meio do SAS (SAS INSTITUTE, 1994). Para testar a hipótese " $b=3,0$ " foi realizado o teste $t$ de Student ao nível de $1 \%$ de significância. Nas relações entre características de unidade de medidas diferentes, se " $b=3,0$ " o crescimento foi denominado isométrico, o que indica que as taxas de crescimento de " $X$ " e "Y" foram semelhantes; se " $b \neq 3,0$ " $o$ crescimento foi considerado alométrico, isto é, o crescimento de uma característica é diferenciado em relação à outra (ROCHA et al., 2002).

\section{RESULTADOS E DISCUSSÃO}

De acordo com o resumo das análises de variância o tipo de nascimento, idade da mãe ao parto e ano de nascimento foram os fatores que mais afetaram o peso e as medidas corporais dos cordeiros ao nascimento e ao desmame, conforme a Tabela 1.

Todas as mensurações ao nascimento e ao desmame, exceto altura ao desmame foram influenciados pela idade da mãe ao parto, em que filhos de ovelhas mais jovens (dois dentes) apresentaram médias menores (Tabela 2 e 3 ).

Tabela 1. Resumos das análises de variâncias dos pesos e medidas corporais ao nascimento e ao desmame e dos ganhos médios diários dessas características

\begin{tabular}{|c|c|c|c|c|c|c|c|}
\hline \multirow{2}{*}{ Fonte de variação } & \multirow{2}{*}{$\begin{array}{l}\text { Graus de } \\
\text { liberdade }\end{array}$} & \multicolumn{6}{|c|}{ Quadrados médios } \\
\hline & & $\mathrm{PN}$ & $\mathrm{PD}$ & GMP & PTN & PTD & GMPT \\
\hline Idade da Mãe ao Parto & 3 & $1,81^{*}$ & $27,29 *$ & 0,004 & $25,75^{* *}$ & $46,47 *$ & 0,007 \\
\hline Tipo de Nascimento & 1 & $16,13^{*}$ & $495,39 * *$ & $0,067 * *$ & $164,1 * *$ & $1113,98 * *$ & $0,086^{* *}$ \\
\hline Grupo Genético & 1 & $2,81^{*}$ & 0,33 & - & 21,23 & 5,61 & 0,009 \\
\hline Sexo do Cordeiro & 1 & $2,91 *$ & $58,72 *$ & 0,007 & $48,39 * *$ & $86,21^{*}$ & 0,001 \\
\hline Ano de Nascimento & 2 & $4,63 *$ & $153,05 * *$ & $0,021 * *$ & $17,44^{*}$ & $554,79 * *$ & $0,082 * *$ \\
\hline Peso da Mãe ao Parto & 1 & $2,25 * *$ & $212,83 * *$ & $0,034 * *$ & 6,37 & $370,40 * *$ & $0,057 * *$ \\
\hline Resíduo & 125 & 0,37 & 9,97 & 0,002 & 6,37 & 14,50 & 0,003 \\
\hline \multirow{2}{*}{ Fonte de variação } & Graus de & \multicolumn{6}{|c|}{ Quadrados médios } \\
\hline & liberdade & $\mathrm{CN}$ & $\mathrm{CD}$ & GMC & $\mathrm{AN}$ & $\mathrm{AD}$ & GMA \\
\hline Idade da Mãe ao Parto & 3 & $22,22 * *$ & $30,08^{*}$ & 0,006 & $21,65 * *$ & 37,88 & 0,003 \\
\hline Tipo de Nascimento & 1 & $71,61 * *$ & $428,39 * *$ & $0,030 * *$ & $99,84 * *$ & $356,89 * *$ & $0,016^{*}$ \\
\hline Grupo Genético & 1 & $33,02 * *$ & 1,23 & 0,009 & $34,36 * *$ & 20,52 & - \\
\hline Sexo do Cordeiro & 1 & 9,58 & 33,95 & 0,001 & $35,61 * *$ & $88,64^{*}$ & 0,002 \\
\hline Ano de Nascimento & 2 & $105,52 * *$ & $2040,58 * *$ & $0,248 * *$ & 3,08 & 41,4 & $0,012 *$ \\
\hline Peso da Mãe ao Parto & 1 & 5,41 & $190,76^{* *}$ & $0,026^{* *}$ & 8,35 & $176,96^{* *}$ & $0,022 * *$ \\
\hline Resíduo & 125 & 4,52 & 10,40 & 0,002 & 4,36 & 15,08 & 0,003 \\
\hline
\end{tabular}

$\mathrm{PN}=$ peso ao nascer; $\mathrm{PD}=$ peso ao desmame; $\mathrm{GMP}=$ ganho médio diário de peso; $\mathrm{PTN}=$ perímetro torácico ao nascer; $\mathrm{PTD}=$ perímetro torácico ao desmame; GMPT $=$ ganho médio diário de perímetro torácico; $\mathrm{CN}=$ comprimento ao nascer; $\mathrm{CD}=$ comprimento ao desmame; $\mathrm{GMC}=$ ganho médio diário de comprimento; $\mathrm{NA}=$ altura ao nascer; $\mathrm{AD}=$ altura ao desmame; $\mathrm{GMA}$ =ganho médio diário de altura. $* * \mathrm{P}<0,01 ; * \mathrm{P}<0,05$. 
Tabela 2. Médias e erros-padrão do peso e perímetro torácico de cordeiros ao nascimento e ao desmame e dos ganhos médios diários dessas características, de acordo com a idade da mãe ao parto, tipo de nascimento, sexo do cordeiro, grupo genético e ano de nascimento

\begin{tabular}{|c|c|c|c|c|c|c|c|}
\hline Característica & $\mathrm{N}^{\circ}$ & PN (kg) & PD (kg) & GMP (kg/dia) & PTN (cm) & PTD (cm) & GMPT (cm/dia) \\
\hline \multicolumn{8}{|c|}{ Idade da mãe ao parto } \\
\hline Dois dentes & 26 & $3,34 \pm 0,14^{\mathrm{b}}$ & $12,19 \pm 0,72^{b}$ & $0,12 \pm 0,01$ & $35,85 \pm 0,57^{\mathrm{c}}$ & $55,98 \pm 0,87^{b}$ & $0,28 \pm 0,01$ \\
\hline Quatro dentes & 28 & $3,59 \pm 0,14^{\mathrm{ab}}$ & $13,70 \pm 0,76^{\mathrm{ab}}$ & $0,14 \pm 0,01$ & $36,29 \pm 0,61^{b c}$ & $56,90 \pm 0,92^{\mathrm{ab}}$ & $0,29 \pm 0,01$ \\
\hline Seis dentes & 29 & $3,78 \pm 0,12^{\mathrm{a}}$ & $14,79 \pm 0,62^{\mathrm{a}}$ & $0,15 \pm 0,01$ & $37,77 \pm 0,50^{\mathrm{ab}}$ & $59,16 \pm 0,75^{\mathrm{a}}$ & $0,30 \pm 0,01$ \\
\hline Oito dentes & 67 & $3,94 \pm 0,09^{\mathrm{a}}$ & $13,31 \pm 0,47^{\mathrm{ab}}$ & $0,13 \pm 0,01$ & $37,86 \pm 0,37^{\mathrm{a}}$ & $56,85 \pm 0,56^{\mathrm{b}}$ & $0,27 \pm 0,01$ \\
\hline \multicolumn{8}{|c|}{ Tipo de nascimento } \\
\hline Simples & 107 & $4,08 \pm 0,06^{\mathrm{a}}$ & $15,81 \pm 0,35^{\mathrm{a}}$ & $0,16 \pm 0,00^{\mathrm{a}}$ & $38,28 \pm 0,28^{\mathrm{a}}$ & $60,70 \pm 0,42^{\mathrm{a}}$ & $0,32 \pm 0,01^{\mathrm{a}}$ \\
\hline Gemelar & 43 & $3,24 \pm 0,11^{\mathrm{b}}$ & $11,18 \pm 0,59^{\mathrm{b}}$ & $0,11 \pm 0,01^{\mathrm{b}}$ & $35,61 \pm 0,47^{\mathrm{b}}$ & $53,75 \pm 0,72^{b}$ & $0,25 \pm 0,01^{\mathrm{b}}$ \\
\hline \multicolumn{8}{|c|}{ Sexo do cordeiro } \\
\hline Fêmea & 74 & $3,51 \pm 0,09^{b}$ & $12,82 \pm 0,46^{\mathrm{b}}$ & $0,13 \pm 0,01$ & $36,33 \pm 0,36^{\mathrm{b}}$ & $56,40 \pm 0,55^{\mathrm{b}}$ & $0,28 \pm 0,01$ \\
\hline Macho & 76 & $3,81 \pm 0,08^{\mathrm{a}}$ & $14,17 \pm 0,45^{\mathrm{a}}$ & $0,14 \pm 0,01$ & $37,56 \pm 0,36^{\mathrm{a}}$ & $58,04 \pm 0,54^{\mathrm{a}}$ & $0,29 \pm 0,01$ \\
\hline \multicolumn{8}{|l|}{ Grupo genético } \\
\hline Santa Inês & 102 & $3,82 \pm 0,07^{\mathrm{a}}$ & $13,55 \pm 0,38$ & $0,13 \pm 0,01$ & $37,38 \pm 0,31$ & $57,00 \pm 0,46$ & $0,28 \pm 0,01$ \\
\hline 1/2 Santa Inês & 48 & $3,50 \pm 0,10^{\mathrm{b}}$ & $13,44 \pm 0,54$ & $0,14 \pm 0,01$ & $36,50 \pm 0,43$ & $57,45 \pm 0,65$ & $0,28 \pm 0,01$ \\
\hline \multicolumn{8}{|c|}{ Ano de nascimento } \\
\hline 2008 & 45 & $4,07 \pm 0,12^{\mathrm{a}}$ & $15,97 \pm 0,64^{\mathrm{a}}$ & $0,16 \pm 0,01^{\mathrm{a}}$ & $37,68 \pm 0,51$ & $62,01 \pm 0,78^{\mathrm{a}}$ & $0,34 \pm 0,01^{\mathrm{a}}$ \\
\hline 2009 & 53 & $3,61 \pm 0,09^{b}$ & $12,81 \pm 0,48^{\mathrm{b}}$ & $0,13 \pm 0,01^{\mathrm{b}}$ & $36,98 \pm 0,39$ & $55,03 \pm 0,59^{\mathrm{b}}$ & $0,25 \pm 0,01^{\mathrm{b}}$ \\
\hline 2010 & 52 & $3,30 \pm 0,11^{\mathrm{c}}$ & $11,72 \pm 0,59^{\mathrm{b}}$ & $0,12 \pm 0,01^{\mathrm{b}}$ & $36,17 \pm 0,47$ & $54,63 \pm 0,71^{\mathrm{b}}$ & $0,26 \pm 0,01^{\mathrm{b}}$ \\
\hline
\end{tabular}

$\mathrm{PN}=$ peso ao nascer; $\mathrm{PD}=$ peso ao desmame; $\mathrm{GMP}=$ ganho médio diário de peso; $\mathrm{PTN}=$ perímetro torácico ao nascer; $\mathrm{PTD}=$ perímetro torácico ao desmame; $\mathrm{GMPT}=$ ganho médio diário de perímetro torácico; ${ }^{\mathrm{a}, \mathrm{b}, \mathrm{c}, \mathrm{d}}$ médias seguidas de letras diferentes na coluna diferem significativamente $(\mathrm{P}<0,05)$ entre si. 
Tabela 3. Médias e erros-padrão do comprimento e altura de cordeiros ao nascimento e ao desmame e dos ganhos médios diários dessas características, de acordo com a idade da mãe ao parto, tipo de nascimento, sexo do cordeiro, grupo genético e ano de nascimento

\begin{tabular}{|c|c|c|c|c|c|c|c|}
\hline Característica & $\mathrm{N}^{\circ}$ & $\mathrm{CN}(\mathrm{cm})$ & $\mathrm{CD}(\mathrm{cm})$ & GMC (cm/dia) & $\mathrm{NA}(\mathrm{cm})$ & $\mathrm{AD}(\mathrm{cm})$ & GMA $(\mathrm{cm} / \mathrm{dia})$ \\
\hline \multicolumn{8}{|c|}{ Idade da mãe ao parto } \\
\hline Dois dentes & 26 & $26,34 \pm 0,48^{b}$ & $45,89 \pm 0,73^{b}$ & $0,27 \pm 0,01$ & $37,47 \pm 0,47^{b}$ & $51,57 \pm 0,88$ & $0,20 \pm 0,01$ \\
\hline Quatro dentes & 28 & $27,22 \pm 0,51^{\mathrm{b}}$ & $48,42 \pm 0,78^{\mathrm{a}}$ & $0,30 \pm 0,01$ & $39,17 \pm 0,50^{\mathrm{a}}$ & $54,45 \pm 0,94$ & $0,21 \pm 0,01$ \\
\hline Seis dentes & 29 & $27,25 \pm 0,42^{b}$ & $48,35 \pm 0,64^{\mathrm{a}}$ & $0,30 \pm 0,01$ & $38,48 \pm 0,41^{\mathrm{ab}}$ & $54,28 \pm 0,77$ & $0,22 \pm 0,01$ \\
\hline Oito dentes & 67 & $28,37 \pm 0,31^{\mathrm{a}}$ & $47,56 \pm 0,48^{\mathrm{a}}$ & $0,27 \pm 0,01$ & $39,44 \pm 0,31^{\mathrm{a}}$ & $53,68 \pm 0,58$ & $0,20 \pm 0,01$ \\
\hline \multicolumn{8}{|c|}{ Tipo de nascimento } \\
\hline Simples & 107 & $28,17 \pm 0,23^{\mathrm{a}}$ & $49,71 \pm 0,35^{\mathrm{a}}$ & $0,30 \pm 0,01^{\mathrm{a}}$ & $39,68 \pm 0,23^{\mathrm{a}}$ & $55,46 \pm 0,43^{\mathrm{a}}$ & $0,22 \pm 0,01^{\mathrm{a}}$ \\
\hline Gemelar & 43 & $26,41 \pm 0,40^{\mathrm{b}}$ & $45,40 \pm 0,61^{b}$ & $0,27 \pm 0,01^{\mathrm{b}}$ & $37,60 \pm 0,39^{b}$ & $51,53 \pm 0,73^{\mathrm{b}}$ & $0,19 \pm 0,01^{\mathrm{b}}$ \\
\hline \multicolumn{8}{|c|}{ Sexo do cordeiro } \\
\hline Fêmea & 74 & $27,02 \pm 0,31$ & $47,04 \pm 0,47$ & $0,28 \pm 0,01$ & $38,11 \pm 0,30^{b}$ & $52,66 \pm 0,56^{\mathrm{b}}$ & $0,20 \pm 0,01$ \\
\hline Macho & 76 & $27,57 \pm 0,30$ & $48,07 \pm 0,46$ & $0,29 \pm 0,01$ & $39,16 \pm 0,30^{\mathrm{a}}$ & $54,33 \pm 0,56^{\mathrm{a}}$ & $0,21 \pm 0,01$ \\
\hline \multicolumn{8}{|l|}{ Grupo genético } \\
\hline Santa Inês & 102 & $27,84 \pm 0,26^{\mathrm{a}}$ & $47,45 \pm 0,39$ & $0,28 \pm 0,01$ & $39,20 \pm 0,25^{\mathrm{a}}$ & $53,93 \pm 0,47$ & $0,21 \pm 0,01$ \\
\hline 1/2 Santa Inês & 48 & $26,75 \pm 0,36^{\mathrm{b}}$ & $47,66 \pm 0,55$ & $0,29 \pm 0,01$ & $38,08 \pm 0,35^{\mathrm{b}}$ & $53,06 \pm 0,66$ & $0,21 \pm 0,01$ \\
\hline \multicolumn{8}{|c|}{ Ano de nascimento } \\
\hline 2008 & 45 & $29,38 \pm 0,43^{\mathrm{a}}$ & $56,72 \pm 0,66^{\mathrm{a}}$ & $0,39 \pm 0,01^{\mathrm{a}}$ & $38,99 \pm 0,42$ & $52,39 \pm 0,79$ & $0,19 \pm 0,01^{\mathrm{b}}$ \\
\hline 2009 & 53 & $26,27 \pm 0,32^{b}$ & $43,03 \pm 0,49^{b}$ & $0,23 \pm 0,01^{\mathrm{b}}$ & $38,54 \pm 0,32$ & $54,59 \pm 0,60$ & $0,22 \pm 0,01^{\mathrm{a}}$ \\
\hline 2010 & 52 & $26,23 \pm 0,40^{\mathrm{b}}$ & $42,92 \pm 0,60^{b}$ & $0,23 \pm 0,01^{\mathrm{b}}$ & $38,39 \pm 0,39$ & $53,51 \pm 0,73$ & $0,21 \pm 0,01^{\mathrm{ab}}$ \\
\hline
\end{tabular}

$\mathrm{CN}=$ comprimento ao nascer; $\mathrm{CD}=$ comprimento ao desmame; $\mathrm{GMC}=$ ganho médio diário de comprimento; $\mathrm{AN}=$ altura ao nascer; $\mathrm{AD}=\mathrm{Altura}$ ao desmame; GMA= ganho médio diário de altura; ${ }^{\mathrm{a}, \mathrm{b}, \mathrm{c}, \mathrm{d}}$ médias seguidas de letras diferentes na coluna diferem significativamente $(\mathrm{P}<0,05)$ entre si. 
As médias de peso ao nascimento variaram de $3,34 \pm 0,14 \mathrm{~kg}$ nos filhos de ovelhas de dois dentes a $3,94 \pm 0,09 \mathrm{~kg}$ nos filhos de ovelhas de oito dentes, e as médias de peso ao desmame variaram de $12,19 \pm 0,72 \mathrm{~kg}$ nos filhos de ovelhas de dois dentes a 14,79 $\pm 0,62$ nos filhos de ovelhas de seis dentes. Estes resultados concordaram com os obtidos por Mohammadi et al. (2010), em que ovelhas de dois e três anos apresentaram cordeiros mais leves ao nascer e ao desmame em relação às ovelhas mais velhas.

Ribeiro et al. (2008) relataram que devido às ovelhas mais jovens encontrarem-se em crescimento, elas normalmente produzem cordeiros mais leves ao nascimento. Os autores citaram também que em função da menor produção de leite, essas fêmeas produzem cordeiros com menores ganhos de peso e mais leves ao desmame.

Os cordeiros nascidos de partos simples apresentaram maiores médias que os cordeiros gêmeos, para todas as características avaliadas, ao nascimento e ao desmame, conforme as Tabelas $2 \mathrm{e}$ $3)$. As médias de peso ao nascer foram de $3,24 \pm 0,11 \mathrm{~kg}$ a $4,08 \pm 0,06 \mathrm{~kg}$ e para peso ao desmame foram de 11,18 \pm $0,59 \mathrm{~kg}$ a $15,81 \pm 0,35 \mathrm{~kg}$, em que as médias menores foram dos cordeiros gêmeos e as maiores dos cordeiros nascidos como simples.

Rocha et al. (2009) também encontraram influências do tipo de nascimento sobre o desempenho dos cordeiros do nascimento ao desmame, em que os nascidos de partos simples apresentaram um desenvolvimento maior que os cordeiros nascidos de partos gemelares. A principal razão dos animais que nascem de partos gemelares serem menores e apresentarem desenvolvimento menor no período pré-desmame é a competição intrauterina e posteriormente por leite materno (MOHAMMADI et al., 2010).
Em média, os cordeiros machos são maiores e mais pesados que as fêmeas, esta diferença ocorre principalmente devido ao dimorfismo sexual e a influência hormonal (PACHECO \& QUIRINO, 2008). O efeito sexo do cordeiro influenciou o peso, o perímetro torácico e altura ao nascimento e ao desmame, no qual os machos apresentaram maiores médias que as fêmeas (Tabela 2 e 3 ). $O$ peso médio ao nascimento e ao desmame foi maior nos machos $(3,81 \pm 0,08$ e $14,17 \pm 0,45 \mathrm{~kg}$ respectivamente) que nas fêmeas $(3,51 \pm$ 0,09 e $12,82 \pm 0,46 \mathrm{~kg}$ respectivamente), estes resultados estão de acordo com os obtidos por Rashidi et al. (2008).

$\mathrm{O}$ efeito ano de nascimento influenciou todas as características avaliadas, ao nascimento e ao desmame, de acordo com as Tabelas 2 e 3 . O efeito de ano é importante em todas as fases do crescimento, observado principalmente em animais manejados a pasto (RIBEIRO et al., 2008), assim como no presente estudo. Isto ocorre principalmente devido às variações que são observados ao longo dos anos, das práticas de manejo, de clima, de ambiente e de alimentos disponíveis aos animais (MOHAMMADI et al., 2010).

Os animais puros da raça Santa Inês foram mais pesados ao nascimento e tiveram maiores médias de comprimento e altura ao nascimento que os animais $1 / 2$ Santa Inês $(P<0,05)$, conforme as Tabelas 2 e 3 . Isso provavelmente ocorreu porque a raça Santa Inês é de maior porte que os animais SRD provenientes da Fazenda Escola da UEL. Araújo Filho et al. (2007) em um estudo com cordeiros Santa Inês puros e $1 / 2$ sangue Santa Inês, relataram que os mesmos não apresentaram diferenças significativas quanto às medidas de comprimento $\mathrm{e}$ perímetro torácico, porém os cordeiros puros Santa Inês foram mais altos que 
Rev. Bras. Saúde Prod. Anim., Salvador, v.13, n.1, p.258-270 jan/mar, 2012 http://www.rbspa.ufba.br ISSN 15199940

os cordeiros $1 / 2$ Santa Inês.

O peso da mãe ao parto exerceu efeito linear significativo sobre o peso dos cordeiros ao nascimento e ao desmame, o que concorda com os resultados de Fernandes et al. (2001). O comprimento, $\mathrm{o}$ perímetro torácico e a altura ao desmame, os ganhos médios diários de peso, de comprimento, de perímetro torácico e de altura do nascimento ao desmame também foram influenciados pelo peso da mãe ao parto, e apresentaram comportamento linear (Tabela 4).

Tabela 4. Regressões lineares para peso ao nascimento (PN), peso ao desmame (PD), comprimento ao desmame (CD), perímetro torácico ao desmame (PTD) e altura ao desmame (AD), e ganhos médios diários de peso (GMP), comprimento (GMC), perímetro torácico (GMPT) e altura (GMA), em função do peso da mãe ao parto (PMP)

\begin{tabular}{lcc}
\hline Equações de regressão linear & Coeficiente de determinação $\left(\mathrm{R}^{2}\right)$ & Desvio-padrão \\
$\mathrm{PN}=20,991+0,0338 \mathrm{PMP}$ & 0,57 & 0,64 \\
$\mathrm{PD}=1,1783+0,2423 \mathrm{PMP}$ & 0,97 & 3,58 \\
$\mathrm{CD}=30,5799+0,3066 \mathrm{PMP}$ & 0,91 & 5,90 \\
$\mathrm{PTD}=42,2522+0,2918 \mathrm{PMP}$ & 0,90 & 4,79 \\
$\mathrm{AD}=44,1778+0,1985 \mathrm{PMP}$ & 0,95 & 3,53 \\
$\mathrm{GMP}=-0,0132+0,0030 \mathrm{PMP}$ & 0,96 & 0,05 \\
$\mathrm{GMC}=0,0844+0,0036 \mathrm{PMP}$ & 0,70 & 0,06 \\
$\mathrm{GMPT}=0,1136+0,0032 \mathrm{PMP}$ & 0,89 & 0,06 \\
$\mathrm{GMA}=0,1121+0,0020 \mathrm{PMP}$ & 0,83 & 0,08 \\
\hline
\end{tabular}

As correlações fenotípicas entre as características avaliadas foram positivas e significativas $(\mathrm{P}<0,01)$, exceto a correlação entre o comprimento ao nascimento e a altura ao desmame que não foi significativa $(\mathrm{P}>0,01)$, de acordo com a Tabela 5. A medida corporal que apresentou maior correlação com o peso foi o perímetro torácico, tanto ao nascer como ao desmame, cujos coeficientes de correlação foram 0,75 e 0,86 , respectivamente. $\mathrm{O}$ ganho médio diário de peso apresentou maiores correlações com as mensurações ao desmame do que ao nascimento, principalmente com o peso $(0,98)$ e com o perímetro torácico $(0,83)$.

Vários estudos encontraram correlações altas e positivas entre o peso vivo e as mensurações corporais, como os de Costa Júnior et al. (2006), Menezes et al. (2008) e Reis et al. (2008). Segundo esses trabalhos a medida corporal que apresenta maior correlação com o peso é o perímetro torácico, e consequentemente é a mensuração que melhor prediz o peso, isto porque essas características variam no mesmo sentido, principalmente por as duas apresentarem uma grande relação com o volume do corpo (Tabela 5).

Foram testadas equações de regressões lineares demonstrados na Tabela 6 e não lineares de Huxley (1932) na Tabela 7 para predizer o peso vivo por meio das mensurações corporais. As medidas ao nascimento explicaram em parte o peso vivo ao nascimento, e não foram eficientes para predizer o peso vivo ao 
Rev. Bras. Saúde Prod. Anim., Salvador, v.13, n.1, p.258-270 jan/mar, 2012 http://www.rbspa.ufba.br ISSN 15199940

desmame. As mensurações ao desmame explicaram muito bem o peso vivo ao desmame. A medida corporal que apresentou os melhores coeficientes de determinação $\left(\mathrm{R}^{2}\right)$ foi $\mathrm{o}$ perímetro torácico, e isto pode ser explicado devido às altas correlações dessas medidas com o peso corporal.

Tabela 5. Correlações entre pesos e medidas corporais de cordeiros ao nascimento e ao desmame

\begin{tabular}{lcccccccc}
\hline Item & PD & CN & CD & PTN & PTD & AN & AD & GMP \\
PN & $0,59^{* *}$ & $0,65^{* *}$ & $0,56^{* *}$ & $0,75^{* *}$ & $0,61^{* *}$ & $0,72^{* *}$ & $0,45^{* *}$ & $0,45^{* *}$ \\
PD & - & $0,39^{* *}$ & $0,71^{* *}$ & $0,44^{* *}$ & $0,86^{* *}$ & $0,38^{* *}$ & $0,56^{* *}$ & $0,98^{* *}$ \\
CN & - & - & $0,54^{* *}$ & $0,48^{* *}$ & $0,44^{* *}$ & $0,52^{* *}$ & $0,09 \mathrm{NS}$ & $0,30^{* *}$ \\
CD & - & - & - & $0,36^{* *}$ & $0,76^{* *}$ & $0,29 * *$ & $0,22^{* *}$ & $0,68^{* *}$ \\
PTN & - & - & - & - & $0,49^{* *}$ & $0,62^{* *}$ & $0,32^{* *}$ & $0,34^{* *}$ \\
PTD & - & - & - & - & - & $0,40^{* *}$ & $0,49^{* *}$ & $0,83^{* *}$ \\
AN & - & - & - & - & - & - & $0,46^{* *}$ & $0,28^{* *}$ \\
AD & - & - & - & - & - & - & - & $0,53^{* *}$ \\
\hline
\end{tabular}

$\mathrm{PN}=$ peso ao nascer; $\mathrm{PD}=$ peso ao desmame; $\mathrm{PTN}=$ perímetro torácico ao nascer; $\mathrm{PTD}=$ perímetro torácico ao desmame; $\mathrm{CN}=$ comprimento ao nascer; $\mathrm{CD}=$ comprimento ao desmame; $\mathrm{NA}=$ altura ao nascer; $\mathrm{AD}=$ altura ao desmame; $\mathrm{GMP}=$ ganho médio diário de peso.

${ }^{*} * \mathrm{P}<0,01 ; * \mathrm{P}<0,05$.

Tabela 6. Parâmetros de regressões lineares para estimação do peso vivo de cordeiros ao nascimento e ao desmame a partir de mensurações corporais

\begin{tabular}{|c|c|c|c|c|}
\hline Mensurações & Intercepto & $\begin{array}{l}\text { Coeficiente } \\
\text { de regressão }\end{array}$ & $\begin{array}{c}\text { Coeficiente de } \\
\text { determinação }\left(\mathrm{R}^{2}\right)\end{array}$ & Desvio-padrão \\
\hline \multicolumn{5}{|c|}{ Parâmetros de regressão linear para estimação do peso vivo ao nascimento } \\
\hline Perímetro torácico & $-4,05491$ & 0,21087 & 0,56 & 0,74 \\
\hline Comprimento & $-1,47648$ & 0,19375 & 0,42 & 0,71 \\
\hline Altura & $-5,96349$ & 0,25130 & 0,52 & 0,73 \\
\hline \multicolumn{5}{|c|}{ Parâmetros de regressão linear para estimação do peso vivo ao desmame } \\
\hline \multicolumn{5}{|l|}{ Perímetro torácico } \\
\hline Nascer & $-11,06474$ & 0,67475 & 0,20 & 0,77 \\
\hline Desmame & $-22,40290$ & 0,63249 & 0,74 & 0,78 \\
\hline \multicolumn{5}{|l|}{ Comprimento } \\
\hline Nascer & $-3,17040$ & 0,63272 & 0,15 & 3,55 \\
\hline Desmame & $-5,74845$ & 0,42054 & 0,51 & 3,56 \\
\hline \multicolumn{5}{|l|}{ Altura } \\
\hline Nascer & $-14,03796$ & 0,72454 & 0,15 & 4,24 \\
\hline Desmame & $-16,15498$ & 0,56157 & 0,32 & 4,27 \\
\hline
\end{tabular}


Rev. Bras. Saúde Prod. Anim., Salvador, v.13, n.1, p.258-270 jan/mar, 2012 http://www.rbspa.ufba.br ISSN 15199940

Tabela 7. Parâmetros de regressões não lineares para estimação do peso vivo de cordeiros ao nascimento e ao desmame a partir de mensurações corporais

\begin{tabular}{|c|c|c|c|c|}
\hline Mensurações & $\begin{array}{l}\text { Antilogaritmo } \\
\text { "a" }\end{array}$ & $\begin{array}{l}\text { Coeficiente de } \\
\text { regressão }\end{array}$ & $\begin{array}{c}\text { Coeficiente de } \\
\text { determinação }\left(\mathrm{R}^{2}\right)\end{array}$ & Desvio-padrão \\
\hline \multicolumn{5}{|c|}{ Parâmetros de regressão alométrica para estimação do peso vivo ao nascimento } \\
\hline Perímetro torácico & 0,00265 & 2,00462 & 0,56 & 0,73 \\
\hline Comprimento & 0,03112 & 1,44883 & 0,45 & 0,72 \\
\hline Altura & 0,00026 & 2,61339 & 0,54 & 0,74 \\
\hline \multicolumn{5}{|c|}{ Parâmetros de regressão alométrica para estimação do peso vivo ao desmame } \\
\hline \multicolumn{5}{|c|}{ Perímetro torácico } \\
\hline Nascer & 0,02140 & 1,78037 & 0,18 & 3,48 \\
\hline Desmame & 0,00023 & 2,70934 & 0,74 & 4,25 \\
\hline \multicolumn{5}{|l|}{ Comprimento } \\
\hline Nascer & 0,23086 & 1,22933 & 0,13 & 3,43 \\
\hline Desmame & 0,03289 & 1,56286 & 0,52 & 4,07 \\
\hline \multicolumn{5}{|l|}{ Altura } \\
\hline Nascer & 0,00698 & 2,06603 & 0,13 & 3,44 \\
\hline Desmame & 0,00075 & 2,45797 & 0,36 & 3,81 \\
\hline
\end{tabular}

As equações lineares apresentaram resultados muito parecidos com os resultados das equações alométricas expressas nas Tabelas 6 e 7. Existem poucos trabalhos na literatura que testaram as equações não lineares para estimar o peso vivo e, em alguns casos, elas são mais eficientes que as equações lineares. Sowande \& Sobola (2008), em um trabalho com ovinos de 13 a 36 meses de idade afirmaram que as equações alométricas foram mais eficientes para estimar o peso vivo desses animais, que as equações lineares simples.

As regressões lineares múltiplas apresentaram melhores coeficientes de determinação que as regressões simples (Tabela 8). Isto quer dizer que equações que utilizam mais de uma mensuração corporal são mais eficientes para estimar o peso vivo que equações que utilizam somente uma mensuração, o que concorda com resultados encontrados por Reis et al. (2008) e Sowand \& Sobola (2008).
Segundo Carvalho et al. (2007) o crescimento pode ser melhor avaliado por meio de equações exponenciais, nas quais cada unidade pode ser comparada ao crescimento total, e assim identificam-se o crescimento diferencial das partes corpo. A partir das medidas corporais foram determinadas equações de regressões não lineares, para estimar o desenvolvimento do peso corporal (Tabela 9).

A relação entre peso e altura apresentou crescimento alométrico positivo $(\mathrm{P}<0,01)$, com o coeficiente de regressão (b) igual a 3,61. Este resultado indica que o peso aumentou proporcionalmente mais que a altura no período pré-desmame. A relação entre peso e comprimento e peso e perímetro torácico apresentaram crescimento alométrico negativo $(\mathrm{P}<0,01)$, com $\mathrm{b}=2,14$ e $\mathrm{b}=2,81$, respectivamente. Estes resultados indicam que o comprimento e o perímetro torácico aumentaram proporcionalmente mais que o peso no período estudado (Tabela 8). 
Rev. Bras. Saúde Prod. Anim., Salvador, v.13, n.1, p.258-270 jan/mar, 2012 http://www.rbspa.ufba.br ISSN 15199940

Tabela 8. Equações de regressões lineares múltiplas para estimar o peso vivo de cordeiros ao nascimento e ao desmame a partir de mensurações corporais

\begin{tabular}{lcc}
\hline Equações preditivas & $\begin{array}{c}\text { Coeficiente de } \\
\text { determinação }\left(\mathrm{R}^{2}\right)\end{array}$ & $\begin{array}{c}\text { Desvio- } \\
\text { padrão }\end{array}$ \\
\hline $\mathrm{PN}=-5,2668+0,1606 \mathrm{PTN}+0,1116 \mathrm{CN}$ & 0,68 & 0,77 \\
$\mathrm{PN}=-7,1709+0,1182 \mathrm{PTN}+0,0846 \mathrm{CN}+0,1082 \mathrm{AN}$ & 0,73 & 0,78 \\
$\mathrm{PD}=-15,1226+0,5064 \mathrm{PTN}+0,3737 \mathrm{CN}$ & 0,24 & 3,55 \\
$\mathrm{PD}=-18,5082+0,4310 \mathrm{PTN}+0,3256 \mathrm{CN}+0,1924 \mathrm{AN}$ & 0,25 & 3,56 \\
$\mathrm{PD}=-28,7044+0,5633 \mathrm{PTD}+0,1894 \mathrm{AD}$ & 0,77 & 4,24 \\
$\mathrm{PD}=-29,1843+0,4198 \mathrm{PTD}+0,1319 \mathrm{CD}+0,2351 \mathrm{AD}$ & 0,79 & 4,27 \\
\hline
\end{tabular}

$\mathrm{PN}=$ peso ao nascer; $\mathrm{PD}=$ peso ao desmame; $\mathrm{PTN}=$ perímetro torácico ao nascer; $\mathrm{PTD}=$ perímetro torácico ao desmame; $\mathrm{CN}=$ comprimento ao nascer; $\mathrm{CD}=$ comprimento ao desmame; $\mathrm{NA}=$ altura ao nascer; $\mathrm{AD}=$ altura ao desmame.

Tabela 9. Valores do antilogaritmo de "a" (Lna), coeficiente de alometria (b), coeficiente de determinação $\left(\mathrm{R}^{2}\right)$ e tipo de crescimento para as relações entre as características: peso e comprimento; peso e perímetro torácico; e peso e altura, em cordeiros do nascimento ao desmame

\begin{tabular}{lccccc}
\hline Item & $\mathrm{N}^{\circ}$ & Lna & $\mathrm{b}$ & $\mathrm{R}^{2}$ & Tipo de crescimento \\
\hline Comprimento & 900 & $-5,6358$ & 2,1394 & 0,85 & Alométrico -** \\
Perímetro torácico & 900 & $-8,7854$ & 2,8081 & 0,90 & Alométrico -** \\
Altura & 900 & $-11,7908$ & 3,6107 & 0,79 & Alométrico +** \\
\hline
\end{tabular}

$\mathrm{N}^{\circ}=$ número de observações; ** $\mathrm{P}<0,01$.

Na Figura 1 são apresentadas as equações alométricas do peso em função das medidas corporais e o comportamento das linhas de regressão, onde observa-se que as medidas comprimento $\mathrm{e}$ perímetro torácico apresentaram maior desenvolvimento que o peso corporal, enquanto que a altura apresentou um desenvolvimento mais lento que o peso, o quer dizer que o comprimento e o perímetro torácico apresentam um crescimento precoce e altura um crescimento tardio.

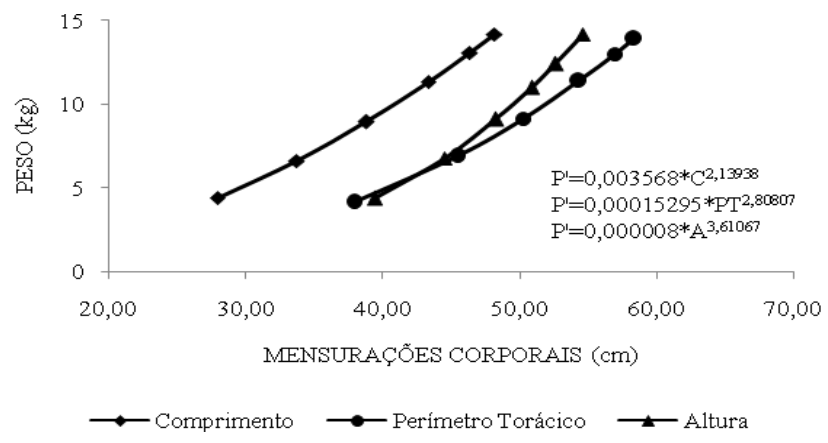

Figura 1. Evolução do peso em relação às medidas corporais de cordeiros do nascimento até os 70 dias de idade 
Rev. Bras. Saúde Prod. Anim., Salvador, v.13, n.1, p.258-270 jan/mar, 2012 http://www.rbspa.ufba.br ISSN 15199940

Com este trabalho foi possível concluir que dentre os principais fatores que afetaram o desenvolvimento dos cordeiros ao nascimento e ao desmame estão o tipo de nascimento, a idade da mãe ao parto e o ano de nascimento. Os pesos e medidas corporais foram todos correlacionados positivamente, e foi possível prever uma por meio da outra através de regressões. A variável que melhor explicou o peso foi $o$ perímetro torácico. O estudo do tipo de crescimento indicou que as características comprimento e perímetro torácico apresentaram maior desenvolvimento que o peso corporal, porém a altura apresentou um desenvolvimento mais lento que o peso, no período estudado.

\section{AGRADECIMENTOS}

Os autores agradecem ao Conselho Nacional de Pesquisa e Desenvolvimento (CNPq) pelas bolsas de produtividade em pesquisa de Ribeiro e Mizubuti.

\section{REFERÊNCIAS}

ARAÚJO FILHO, J.T.; COSTA, R.G.; FRAGA, A.B.; SOUSA, W.H.; GONZAGA NETO, S.; BATISTA, A.S.M.; CUNHA, M.G.G. Efeito de dieta e genótipo sobre medidas morfométricas e não constituintes da carcaça de cordeiros deslanados terminados em confinamento. Revista Brasileira de Saúde e Produção Animal [Online], v.8, n.4, p.394-404, 2007.

CARVALHO, P.A.; SANCHEZ, LM.B.; VELHO, J.P.; PIRES, C.C.; VIEGA, J. Crescimento alométrico de componentes da carcaça de bezerros de origem leiteira na fase inicial do crescimento pós-natal. Ciência Rural, v.37, n.1, p.223-228, 2007.
COSTA JÚNIOR, G.S.; CAMPELO, J.E.G.; AZEVEDO, D.M.M.R.; MARTINS FILHO, R.; CAVALCANTE, R.R.; LOPES, J.B.; OLIVEIRA, M.E. Caracterização morfométrica de ovinos da raça Santa Inês criados nas microrregiões de Teresina e Campo Maior, Piauí. Revista Brasileira de Zootecnia, v.35, n.6, p.2260-2267, 2006.

FERNANDES, A.A.O.; BUCHANAN, D.; SELAIVE-VILLARROEL, A.B. Avaliação dos fatores ambientais no desenvolvimento corporal de cordeiros deslanados da raça Morada Nova.

Revista Brasileira de Zootecnia, v.30, n.5, p.1460-1465, 2001.

GUEDES, M.H.P.; MUNIZ, J.A.; SILVA, F.F.; AQUINO, L.H. Análise Bayesiana da curva de crescimento de cordeiros da raça Santa Inês. Arquivos Brasileiros de Medicina Veterinária e Zootecnia, v.57, n.3, p.415-417, 2005.

HUXLEY, J.S. Problems of relative growth. London: Methuen, 1932. 276p.

MACMANUS, C.; EVANGELISTA, C.; FERNANDES, L.A.C.; MIRANDA, R.M.; MORENO-BERNAL, F.E.; SANTOS, N.R. Curvas de crescimento de ovinos Bergamácia criados no Distrito Federal. Revista Brasileira de Zootecnia, v.32, n.5, p.1207-1212, 2003.

MALHADO, C.H.M.; CARNEIRO, P.L.S.; SANTOS, P.F.; AZEVEDO, D.M.M.R.; SOUZA, J.C.; AFFONSO, P.R.M. Curva de crescimento em ovinos mestiços Santa Inês x Texel criados no Sudoeste do Estado da Bahia. Revista Brasileira de Saúde Produção Animal, v.9, n.2, p. 210-218, 2008. 
Rev. Bras. Saúde Prod. Anim., Salvador, v.13, n.1, p.258-270 jan/mar, 2012 http://www.rbspa.ufba.br ISSN 15199940

MANDAL, A.; ROY, R.; ROUT, P.K. Direct and maternal effects for body measurements at birth and weaning in Muzaffarnagari sheep of India. Small Ruminant Research, v.75, p.123-127, 2008.

MENEZES, L.F.G.; RESTLE, J.; KUSS, F.; BRONDANI, I.L.; ALVES FILHO, D.C.; CATELLAM, J.; OSMARI, M.P. Medidas corporais de novilhos das gerações avançadas do cruzamento rotativo entre as raças Charolês e Nelore, terminados em confinamento. Ciência Rural, v.38, n.3, p.771-777, 2008.

MOHAMMADI, K.; BEYGI NASSIRI, M.T.; FAYAZI, J.; ROSHANFEKR, H. Investigation of environmental factors influence on pre-weaning growth traits in Zandi lambs. Journal of Animal and Veterinary Advances, v.9, n.6, p.10111014, 2010.

PACHECO, A.; QUIRINO, C.R.

Estudo das características de crescimento em ovinos. Pubvet, v.2, n.29, p.1982-1263, 2008.

RASHIDI, A.; MOKHTARI, M.S.; SAFI JAHANSHARI, A.; MOHAMMADI ABADI, M.R. et al. Genetic parameter estimates of preweaning growth traits in Kermani sheep. Small Ruminant Research, v.74, p.165-171, 2008.

REIS, L.R.; ALBIQUERQUE, F.H.M.A.R.; VALENTE, B.D.; MARTINS, G.A.; TEODORO, R.L.; FERREIRA, M.B.D.; MONTEIRO, J.B.N.; SILVA, M.A.; MADALENA, F.E. Predição do peso vivo a partir de medidas corporais em animais mestiços Holandês/Gir. Ciência Rural, v.38, n.3, p.778-783, 2008.
RIBEIRO, E.L.A.; MIZUBUTI, I.Y.; SILVA, L.D.F.; ROCHA, M.A.; SILVA, A.P.; MORI, R.M.; FERREIRA, D.O.L.; CASIMIRO, T.R. Desempenho produtivo de ovelhas submetidas a acasalamento no verão ou no outono no Norte do Paraná. Semina: Ciências Agrárias, v.29, n.1, p.229236, 2008.

ROCHA, L.P.; FRAGA A.B.; ARAÚJO FILHO J.T.; FIGUEIRA, R.F.; PACHECO, K.M.G.; SILVA, F.L.; RODRIGUES, D.S. Desempenho de cordeiros cruzados em Alagoas, Brasil. Archivos de Zootecnia, v.58, n.221, p.145-148, 2009.

ROCHA, M.A.; RIBEIRO; E.L.A.; MIZUBUTI; I. Y.; SILVA; L.D.F. Parâmetros de crescimento e suas correlações em idades entre 60 as 240 dias na carpa húngara (Cyprinos carpio). Semina: Ciências Agrárias, v.23, n.1, p.29-34, 2002.

SANTOS, C.L.; PÉREZ, J.R.O.; MUNIZ, J.A.; GERASEEV1, L.C.; SIQUEIRA, E.R. Desenvolvimento relativo dos tecidos ósseo, muscular e adiposo dos cortes da carcaça de cordeiros Santa Inês. Revista Brasileira de Zootecnia, v.30, n.2, p.487-492, 2001.

\section{SAS INSTITUTE. SAS/STAT User's}

Guide. Cary, 1994. v.2.

SOWAND, O.S.; SOBOLA, O.S. Body measurements of West African Dwarf sheep as parameters for estimation of live weight. Tropical Animal Health and Production, v.40, p.433-439, 2008.

Data de recebimento: 06/05/2011

Data de aprovação: 21/11/2011 